\title{
LA MAESTRA ALDA COSTA: UNA MÁRTIR ANTIFASCISTA
}

\author{
Antonella CAgnOLATI \\ Università degli studi di Foggia \\ E-mail: a.cagnolati@unifg.it
}

Fecha de recepción: 6-III-2011

Fecha de aceptación: 15-IV-2011

RESUMEN: Un sector interesante al que aplicar nuevas categorías de la investigación histórica y social es, sin duda, el de la educación de las mujeres. Maestras, educadoras, pedagogas fueron revolucionarias en diversas épocas de la historia dedicando su existencia a la difusión de conocimientos que pudiesen ser aprovechados por otras mujeres. Para ello fundaron escuelas, escribieron libros y publicaron periódicos. En este sentido las maestras socialistas italianas fueron pioneras al difundir palabras claves como derechos, democracia e igualdad de sueldo. Una figura muy importante fue la maestra antifascista Alda Costa (1876-1944), que luchó contra el militarismo italiano antes de la Primera Guerra Mundial y asumió encargos de gran relevancia en el Partido Socialista. Además fue amiga de Giacomo Matteotti, asesinado por los fascistas en Roma en junio de 1925. Alda Costa fue encarcelada, por su oposición al régimen, y los sufrimientos padecidos precipitaron su muerte. antifascismo

Palabras clave: maestra, derechos de las mujeres, historia de género, educación,

AвSTRACT: A relevant field to apply new theories of historical and social research is the education of women. Teachers, educators and pedagogists were really revolutionary figures in different periods of history, devoting their lives to widespread useful knowledge for other women. In order to do so, they founded schools, they wrote books and started journals. The Italian women of the Socialist Party who worked as teachers were pioneers in propagating key words like rights, democracy, and claiming for equal wages. At the beginning of the XXth century a famous women was the antifascist teacher Alda Costa (1876-1944), who fought against militarism before the First World War. She hold offices of great significance in the Socialist Party, and was also a friend of Giacomo Matteotti, killed 
in Rome by the fascists in 1925. For her opposition to the Mussolini regime Alda Costa was imprisoned, and the cruelties she suffered in jail caused her death.

Key words: teacher, women's rights, gender history, education, antifascism

\section{UNA MUJER VALIOSA}

La necesidad de recuperar en la historia trazas decoloradas nos mueve a ir profundizando para reanimar a los fantasmas de la memoria, los cuales alguna vez se nos presentan tan claros que nos ayudan a investigar nuestro presente. Es la urgencia moral la que me ha convencido de realizar, en ese ensayo, un análisis de la figura de Alda Costa ${ }^{1}$, la cual es considerada por todos, no sólo uno de los personajes más importantes en el socialismo de los primeros años del siglo XX sino, ante todo, una mujer fuerte, valiosa, obstinada antifascista, una educadora que siempre ha planteado su trabajo cotidiano en los ideales que nunca ha abjurado en su atormentada existencia.

$\mathrm{Al}$ igual que otras mujeres que vivieron entre los siglos XIX y XX, Alda recorre la formación profesional, la toma de conciencia política, del pragmatismo organizativo, y de la educación del pueblo para la toma de conciencia de sus derechos. En esa incansable actividad, su visión ética se amplía y se específica hasta convertirse en fuerza propulsiva para cambiar la sociedad y para afirmar con lucidez las maldades del Fascismo, que iban imponiéndose en el territorio de la ciudad de Ferrara con dinámicas

1 Alda Costa nació en Ferrara el 26 de enero de 1876. Hija de Vincenzo y Caterina Zaballi. Su madre, maestra de la escuela primaria, les dirigió tanto a ella como a sus hermanos, Alessandro y Amelia, a los estudios de magisterio. Tras obtenter el diploma, Alda estuvo de suplente en numerosas localidades de la Provincia de Ferrara. El 1 de septiembre de 1899 fue nombrada maestra de la escuela primaria por el Consejo Municipal de la Ciudad de Ferrara. Fue miembro activo del Partido Socialista y se comprometió en la propaganda política, en los mitines y en los artículos de prensa. El Fascismo le dió en el blanco con ataques en la prensa y con verdaderas represalías físicas (aceite de ricino y golpes). En 1926 fue condenada a dos años de destierro en las Islas Tremiti y luego en la Región de Basilicata. Después de haber vuelto a Ferrara, fue encarcelada en la "larga noche" de 1943 (14 de septiembre) y murió el 30 de abril de 1944. Para una síntesis biográfica véase ALESSANDRO ROVERI, "Costa Alda", en Dizionario Biografico degli Italiani, vol. 30, Roma, Istituto della Enciclopedia Italiana, 1984, pp. 124-126; ALESSANDRO ROVERI, "Costa Alda”, en FRANCO ANDREUCCI, TOMMASO DETTI, (a cargo de), Il movimento operaio italiano. Dizionario biografico, Roma, Ed. Riuniti, 1976, vol. II, pp. 106-109; ALFREDO TALAMINI, Alda Costa. La grande perseguitata. Suo calvario. Suoipensieri e scritti, s.l., s.n.; AUTUNNO RAVÀ, Alda Costa. Educatrice, combattente, martire. Trent'anni di stolta persecuzione poliziesca, Ferrara, Partito Socialista Italiano, 1952. Véase tambien ANTONELLA CAGNOLATI, "La professionalità, la politica: Alda Costa", en ANTONELLA CAGNOLATI (a cargo de), Maternità militanti. Impegno sociale tra educazione ed emancipazione, Roma, Aracne, 2010, pp. 115-129. 
políticas y movimentistas que pronto le convirtieron en un poder totalizante y hegemónico².

Alda Costa fue una "de las mujeres que más actuaron con lucidez a nivel político y que el socialismo Italiano pudo expresar" y (me da pena decirlo) de la cual no se encuentra hoy ninguna biografía completa que no la disminuya a "débil maestrita", como se le representa en el fascículo del registro judicial de la Jefatura de policía, que ha sido recientemente adquirido por el Archivo Histórico de la ciudad de Ferrara y cuyo análisis ha sido empezado por Delfina Tromboni. Como el historiador Paul Corner ha señalado bien, Alda fue "uno de los más importantes personajes del socialismo" de la Provincia de Ferrara y según Alessandro Roveri ella fue "el más iluminado y lúcido dirigente socialista en Ferrara"s. Por consiguiente fue mucho más que una simple maestrita! ${ }^{6}$

Más allá de una simple hagiografía popular-patriótica, quiero encontrar en los escritos de Alda Costa una parábola que ponga en sintonía sus elecciones personales con los ideales que ella misma difundió a través de sus palabras. Pues objeto privilegiado de la investigación son sus artículos, siempre precisos y rigurosos en su análisis actual, y sus relatos en los congresos del partido donde se percibe una vis polémica indudablemente muy poco usual en esa época. Como nos informa Talamini, los fascistas afirmaban que "nuestros adversarios se atricheraban detrás de una débil mujer": aquella era Alda7.

Me parece oportuno destacar, como en filigrana, a través de su biografía, en una mezcla sinérgica de microhistoria local y macrohistoria,

2 Sobre el origen del Fascismo en la Provincia de Ferrara véase PAUL R. CORNER, Il Fascismo a Ferrara. 1915-1925, Bari, Laterza, 1974; RENATO SITTI, LUCILLA PREVIATI, Ferrara. Il regime fascista, Milano, La Pietra, 1976.

3 GIUSEPPE INZERILLO, La maestra Alda Costa "vedetta sovversiva", en "Problemi della pedagogia", n. II, julio-diciembre 1976, pp. 1131-1136; GIUSEPPE INZERILLO, Una vedetta "sovversiva": Alda Costa, en WALTER MORETTI (a cargo de), La cultura ferrarese fra le due guerre mondiali, Bologna, Cappelli, 1980, pp. 49-58.

4 PAUL R. CORNER, Il Fascismo a Ferrara, op. cit., p. 112.

5 ALESSANDRO ROVERI, Le origini del Fascismo a Ferrara 1918-1921, Milano, Feltrinelli, 1974, pp. 43 ss.

6 DELFINA TROMBONI, Donne di sentimenti tendenziosi. Sovversive nelle schedature politiche del Novecento, Ferrara, Nuove Carte, 2006. La ficha sobre Alda Costa se encuentra en la página 100.

7 ALFREDO TALAMINI, op. cit., p. 19. 
los atormentados acontecimientos del socialismo en Ferrara ${ }^{8}$ e Italia de esa época, la subida del Fascismo, el sometimiento de la escuela al régimen y a los fines de la propaganda fascista, la confusión y la desorientación después, el 25 de julio de 1943. Lo que resulta verdaderamente interesante es la identidad política de Alda, su clarividencia, sus elecciones de las cuales querría hablar.

Alda empezó su carrera como muchas otras mujeres, entre los últimos años del siglo XIX y los primeros decenios del XX, trabajando como maestra municipal ${ }^{9}$. Pero las persuasivas serenas del socialismo pronto la fascinaron y la llevaron a reconsiderar (y eso es para mí muy importante) las finalidades de su oficio y la huella ética que tenía que atribuirle ${ }^{10}$. Sus repetidas "insubordinaciones", en el desarrollo de su profesión, le costaron la destitución (ilegal) y contribuyeron a fortalecer las acusaciones contra ella, cuando las autoridades locales decidieron condenarla al destierro como "subversiva".

Otro trato característico de su vida fue el periodismo. No solamente se encuentran sus escritos, agudos y penetrantes, en las principales cabeceras de prensa socialista sino que Alda también aportó su contribución como redactora, incitando tomas de posiciones, lanzando advertencias desde los periódicos, reflexionando sobre hechos y acontencimientos de toda la comunidad de Ferrara.

Su colaboración con "Il Pensiero Socialista", el periódico que interpreta el pensamiento del socialismo reformista, empezó en 1905: aunque su estilo revele algo sentimental y a veces retórico, los contenidos

8 ALESSANDRO ROVERI, Socialismo e sindacalismo nel Ferrarese (1870-1915), en "Annuario dell'Istituto Storico Italiano per l'età moderna e contemporanea", voll. XV y XVI (19631964), Roma, s.n., 1968, pp. 150-448; MAURIZIO RIDOLFI, Il Psi e la nascita del partito di massa. 1892-1922, Roma-Bari, Laterza, 1992.

9 Sobre la relevancia de la profesión de maestra véase GIORGIO BINI, Romanzi e realtà di maestri e maestre, en Storia d'Italia, Annali, vol. IV, Intellettuali e potere, Torino, Einaudi, 1981, pp. 1195-1224; SIMONETTA SOLDANI, (a cargo de), L'educazione delle donne, Milano, Angeli, 1991; SIMONETTA SOLDANI, Nascita della maestra elementare, en SIMONETTA SOLDANI, GABRIELE TURI (a cargo de), Fare gli italiani. Scuola e cultura nellitalia contemporanea, Bologna, Il Mulino, 1993, pp. 67-130; SIMONETTA SOLDANI, Maestre d'Italia, en ANGELA GROPPI (a cargo de), Il lavoro delle donne, Roma-Bari, Laterza, 1996, pp. 368-397; CARMELA COVATO, Un'identità divisa. Diventare maestra in Italia tra Otto e Novecento, Roma, Archivio Guido Izzi, 1996.

10 GIUSEPPE BARDELLINI, Alda Costa, en GIUSEPPE BARDELLINI, Socialismo ferrarese. Note sulle prime lotte operaie dall'avvento del Fascismo fino ai giorni nostri, Bologna, A. Brunelli, 1963, pp. 21-29; ENZO SANTARELLI, Alda Costa, en Protagoniste femminili del primo Novecento, en "Problemi del Socialismo", n. 4, cuarta serie, a. XVII, octubre-diciembre 1976, p. 239. 
de sus artículos parecen densos, ricos de inspiraciones políticas aun cuando trazan los acontecimientos cotidianos.

Así, las malas condiciones de los niños, que Alda podía observar cada día, se convertían, a menudo, en objeto de unas reflexiones cargadas de rabia y de pena. En su agudo artículo Refezione, teatro e scheda, Alda, muy expresamente y sin algún artificio retórico, censuraba la decisión de la Municipalidad de Ferrara de conceder fondos para administrar el teatro, un lujo para pocos "holgazanes y muy ricos [que] necesitan pasar muy bien su tiempo libre, y no quieren pagarlo totalmente" ${ }^{11}$. Los mismos fondos habrían producido efectos muy diferentes si hubiesen sido otorgados a los niños para las comidas escolares :

Los veo delante de todos, niños pobrecitos, flacos y harapientos, hambrientos y cansados [...]. Partid para ellos el pan de la ciencia, se nos dice: crueles! Pero ante todo es pan verdadero, bueno y blanco que necesitan y no lo de la ciencia! Enseñad a ellos cogniciones útiles, se nos repite. Ob! Pues, no sabéis que sus débiles mentes de hambrientos no son capaces de coger hasta la más simple de las cogniciones. Qué pena para vosotros los niños la educación pública gratuita y obligatoria. Qué pena y crueldad! Facilitaros un libro cuando en vuestros ojos, en vuestra cara, en todo vuestro pobre cuerpecito solamente queréis gritar: "tengo hambre!'. Daros un boligrafo cuando en el estremecimiento de vuestros cuerpos flacos y aterridos vos gritáis: "tengo frío!"’12.

Se encuentra en muchos de sus escritos, una verdadera solidaridad humana, un deseo de rescate que inflama sus palabras, que produce indignación y voluntad de difundir esa abominación: un estilo lleno de lágrimas y sangre que se mezcla con una vena mesiánica siempre presente en $\mathrm{Alda}^{13}$. Antes que el periódico fuera entregado al grupo maximalista en julio de 1906, otro artículo traza una situación que ella sentía mucho. Se habla de Figure Dolorose y se cuenta la historia de un niño que había sido abandonado por su madre trás ser brutalmente golpeada una vez más. La tarea de los padres habia sido muchas veces desaprobada por Alda Costa la cual veía en su ignorancia el obstáculo más difícil de superar para el destino de sus pequeños alumnos. Con gran frecuencia ella hablaba de deberes de los padres, en una sociedad campesina patriarcal donde la voluntad del padre era ley para el hijo, incitándoles a pensar en un futuro más justo para sus hijos uniéndose a ligas y haciendo huelga por una causa justa ${ }^{14}$.

11 ALDA COSTA, Refezione, teatro e scheda, "Il Pensiero Socialista", 24 de febrero de 1906.

\section{Ibidem.}

13 Sobre el estilo y los contenidos de los escritos femeninos en los periódicos del mismo período, concédame remitir a SILVIA FRANCHINI, SIMONETTA SOLDANI (a cargo de), Donne e giornalismo. Percorsi e presenze di una storia di genere, Milano, F. Angeli, 2004; ANTONELLA CAGNOLATI, TIZIANA PIRONI, Cambiare gli occhi al mondo intero. Donne nuove ed educazione nelle pagine de L'Alleanza (1906-1911), Milano, Unicopli, 2006.

14 ALDA COSTA, Per essi, "Il Pensiero Socialista”, 3 de marzo de 1906. 
Apólogos tristes y acusadores que saltan fuera de las páginas: véase el cuento Quel giorno egli odio ${ }^{15}$ o Né la culla né la tomba ${ }^{16}$, historia de un comienzo precoz hacia la corrupción del cuerpo y del alma de dos niñas pequeñas que bailan por la calle mendicando, guiñando como mujeres perdidas.

Uno de los temas que más se ha tratado en los artículos escritos para "La Bandiera Socialista" 17 es, sin duda, el debate del Partido Socialista entre reformistas y maximalistas, del cual nos informan muy ampliamente a través de las páginas del periódico, donde también se encuentran los relatos en los congresos y en las asambleas de los ciudadanos. En hechos de dimensión limitadamente local se sumaron acontecimientos de gran relevancia histórica, como la guerra en Libia que obligó a los miembros del Partido a tomar una posición, y apoyar a menudo facciones opuestas. Si unos reformistas aceptaron la guerra, otros se declararon abiertamente en contra: entre ellos estaba Alda Costa que, incansable, escribió en favor del pacifismo hasta llegar a tomar una posición tan radical que levantó críticas también dentro de su mismo partido, como ocurrió unos años más tarde con el debate entre los interventisti (que creían en la intervención de Italia en la guerra) y los neutralisti (que rechazaban la guerra), el cual abrió las puertas a la propaganda de Mussolini. Se pueden leer palabras enérgicas en Sventura e colpa donde Alda denuncia el cinismo difuso de "unos hombres, todavía dueños de la vida y de la muerte de los demás"18, que obliga a una nación entera a la lucha sembrando luto, muerte y ruina, para ir detrás de "su gran hado", y la conjura del silencio que ha hecho callar a los periódicos para cantar "hosanna a la concordia nacional".

Una Casandra inescuchada, una profetisa sin desgracias: tal parecía en esa época Alda Costa que no se cansaba de ser el alma crítica del socialismo en Ferrara, ya en poder de los diablos tentadores del sindicalismo revolucionario. Alda traza con sorprendente clarividencia lo que irá a pasar: el frente socialista partido, las luchas fratricidas, la propaganda en las plazas para la entrada en guerra en 1915. Es, en esa desesperación, el anuncio de

15 ALDA COSTA, Quel giorno egli odiò, "Il Pensiero Socialista", 31 de marzo de 1906.

16 ALDA COSTA, Né la culla né la tomba, "Il Pensiero Socialista", 14 de abril de 1906.

17 Sobre las vicisitudes del periódico véase VIOLETTA FERRIOLI, La Bandiera Socialista, en ALDO BERSELLI, 1892-1992. Il movimento socialista ferrarese dalle origini alla nascita della repubblica democratica: contributi per una storia, Cento, Centoggi, 1992, pp. 219-221. El primer número del semanario fue publicado el 10 de enero de 1914. En la redacción había Alda Costa. Véase también ANNA MARIA QUARZI, La condizione della donna nella stampa periodica ferrarese del primo Novecento. Da "Bandiera Socialista" a "Corriere padano", en WALTER MORETTI, op. cit., pp. 99-103, y ANNA MARIA QUARZI, ANNAMARIA ANSALONI, L'azione politica e formativa di Alda Costa, en ILVA VACCARI (a cargo de), La donna nel ventennio fascista 1919-1943, Milano, Vangelista, 1978, pp. 305-317.

18 ALDA COSTA, Sventura e colpa, "La Bandiera Socialista", 24 de enero de 1915. 
tiempos más y más sombríos descritos con amarga lucidez en artículos "gritados", para denunciar el diluvio inminente causado por el silencio de las conciencias Europeas: un artículo que derrama lágrimas, cuyo título emblemático Contro tutti gli imperialismi ${ }^{19}$ nos hace tomar una posición fuerte contra todos los imperialismos, contra los cuales los trabajadores unidos tendrían que oponerse.

En el mismo sentido fue el relato político que Alda expuso en el Congreso del Partido del 28 de febrero de 1915, como miembro de la secretaría: después de exhaustivas referencias a la situación local, ella terminó manifestando su intransigente posición neutralista y "su actitud de lucha sin parar contra la invasión de la tendencia interventista" ${ }^{20}$. Su trabajo incansable se había concretado en la manifestación del 21 de febrero "cuyo éxito fue maravilloso y demostró una indomable aversión de los socialistas y de los trabajadores al ingreso de Italia en la guerra" ${ }^{21}$. El contenido de la obra de Alda y de toda la secretaría suscitó consentimientos unánimes ${ }^{22}$. Lamentablemente los hechos de los meses siguientes contradijeron el optimismo, como aparece muy claramente en unos artículos publicados entre marzo y agosto de 1915.

\section{LA MAESTRA "SUBVERSIVA"}

En noviembre de 1916 Alda Costa participó en el Congreso regional socialista en Bologna y fue nombrada por la asamblea responsable por la Provincia de la ciudad de Ferrara de la propaganda pacifista y de la organización femenina del Partido. Costa desempeñó un papel importante en las agitaciones antimilitaristas de las mujeres que tuvieron lugar en Barra, Copparo, en el Argentano y en el Codigorese, desde el mes de enero de 1917. La propaganda se encontraba así tan extensa y ramificada que Giuffrida, el prefecto de Ferrara, aún rechazando la posibilidad de la existencia de un verdadero subcomité revolucionario, informó al Ministerio del Interior que según él, si la palabra de orden de la revolución socialista hubiese llegado, en la Provincia de Ferrara habría sido pronta la respuesta, remarcando como entre los "violentos" estaba también Alda Costa. El activismo de

19 ALDA COSTA, Contro tutti gli imperialismi, "La Bandiera Socialista", 21 de febrero de 1915.

20 ALDA COSTA, Relazione politica della Federazione provinciale socialista, "La Bandiera Socialista", 7 de marzo de 1915.

\section{Ibidem.}

22 "El Congreso aprueba unánime el largo y lúcido relato político de la secretaria Alda Costa, la cual da las gracias por la actividad incansable y el amor hacia el Partido; y reafirma como justa, proficua y correspondiente a la dirección del Partido la táctica perseguida por la Federación Socialista", Ibidem. 
Alda resultaba ser peligroso: muy temprano llegó la ficha que llevaba su nombre: "la subversiva Alda Costa tiene en la opinión pública mala fama por su manera de ser altanera, conduce una vida privada equívoca hasta considerarla una mujer cuyas costumbres son de procedencia incierta" ${ }^{23}$.

La ficha marcó el comienzo de las persecuciones y un interés patológico de la prensa (cierta prensa) hacia ella. El momento crucial llegó en 1917: desde entonces los ideales políticos y la práctica cotidiana más se unieron en la vida de Alda Costa.

"Il Fascio" destinó dos amplios artículos a Alda Costa: en el primero le atacaba por su fe socialista; en el segundo contaba, a su manera, el episodio del cine subrayando como todos los alumnos habían sido acompañados a la proyección "menos aquellos que tienen por suerte una maestra socialista: Alda Costa" 24 .

"La Provincia de Ferrara"25 iba comentando en un artículo, desde el título clarísimo L'atteggiamento antipatriottico di un'insegnante, marcando el gesto de Alda como pura propaganda antipatriótica, e iba invitando a las autoridades para que reprimieran actos similares porque eran perjudiciales al interés de la patria. A su total disculpa, Alda escribió una carta que fue publicada en "La Bandiera Socialista" (y que la "Gazzetta ferrarese" no había querido publicar), en la cual ella aclaraba las razones de su gesto. Contra una escuela donde pasan mensajes violentos y se fomenta la guerra y la sangre, Alda manifestaba su idea de escuela y de educación:

en el gesto por mi hecho se encuentra la reacción a toda esa dirección educativa y también la aserción que la escuela tiene que ser humana, universal y que no suscite odios y deseos de venganza. Y además tiene ante todo, albergando en una área de serenidad y calma y sin ser perturbada por los ecos de las matanzas y de las ruinas, que generar sentidos que acaso algún día gobernarán el mundo ${ }^{26}$.

Desde entonces Alda siempre fue más vigilada. Su actividad política continuó pero el ala reformista del Partido Socialista en Ferrara se encontraba claramente en minoría: se esperaba la revolución y los maximalistas ya se consideraban triunfantes. Alda sentía lucidamente el peligro de una separación y los daños inestimables de sus posibles repercusiones. Muchas intervenciones, en particular durante las huelgas del 1920, indicaban la necesidad de seguir con el acción de información y propaganda, de

23 Cit. en AUTUNNO RAVÀ, op. cit., p. 9, donde se encuentran fragmentos sacados de la ficha de Alda Costa.

24 Los artículos fueron publicados en "Il Fascio" el 1 de abril de 1917.

25 L'atteggiamento antipatriottico di un'insegnante, "La Provincia di Ferrara", 27 de marzo de 1917.

26 ALDA COSTA, Un problema di educazione, en "La Bandiera Socialista”, 1 de abril de 1917. 
sensibilización de los trabajadores. Artículos en "La Scintilla"27 destacan la lucidez política a través de la cual Alda miraba la situación: el partido tendría que elegir entre reforma y revolución.

Su clarividencia no pudo evitar el desastre: desde el 1920 empezaron las acciones de las patrullas fascistas en los campos alrededor de Ferrara, la situación se hizo tensa hasta el acontecimiento del 20 de diciembre de 1920, cuando en una pelea violenta con ocasión del Consejo provincial socialista murieron tres fascistas y dos socialistas ${ }^{28}$. Las trazas sobre Alda se hacen más lábiles y evanescentes en ese período: sabemos que el Fascismo se encarnizó contra ella (aceite de ricino y otras varias amenidades de camerada), que ella se ocupó de las cuestiones sindicales de su categoría como, por ejemplo, la igualdad salarial entre maestros y maestras.

Pero el hacha del régimen también se abatió con violencia sobre ella, vieja amiga de Giacomo Matteotti. En junio de 1925 un nuevo aviso del prefecto de Ferrara informa que Alda "nunca se ha cansado de hacer propaganda subversiva en favor de las organizaciones de los trabajadores e interesándose por los presos políticos [...] participa en asambleas y encuentros que convoca y organiza. Recibe cartas subversivas del Reino y del extranjero y está considerada tan peligrosa para el orden público que ella está vigilada" 29 .

\section{EL DESTIERRO Y LA MUERTE}

El21 de diciembre de 1925 a ella le pidieron ajustarse a las disposiciones del saludo romano; Alda se negó, motivando su decisión en una carta al alcalde de la ciudad de Ferrara ${ }^{30}$. Se sometió al juramento con tal reserva que fue obligada (después de una denuncia de su comportamiento desdeñoso en el "Corriere padano" de Italo Balbo) a repetirlo. El régimen quería librarse de ella: sufrió una inspección y un duro interrogatorio. El material que encontraron en su casa fue suficiente para incriminarla: el alcalde la denunció al Prefecto (Bertini, ex Comisario de Roma, premiado por encubrir los asesinos de Matteotti), que despidió a la maestra con un acto del 7 de agosto

27 Sobre las posiciones políticas manifestadas en el periódico véase PAOLA DE PAOLI, La Scintilla, en ALDO BERSELLI, op. cit., pp. 87-90.

28 ROBERTO VIVARELLI, Storia delle origini del Fascismo. L'Italia dalla grande guerra alla marcia su Roma, Bologna, Il Mulino, 1991, vol. II, pp. 328-364, y 806-857.

29 GIUSEPPE INZERILLO, La maestra Alda Costa "vedetta sovversiva", cit., p. 1135.

30 ARCHIVIO STORICO COMUNALE DI FERRARA (desde ahora ASCFe), sobre 25, fasc.I, Causa Alda Costa-Municipio di Ferrara, carta manuscrita por la maestra al Alcalde de Ferrara, 22 de diciembre de 1925. 
de 1926 "por abierta y explícita profesión de fe socialista"31, aunque ese acto fuera manifestadamente ilegal como afirmó el Consejo de la disciplina de Emilia Romagna.

Todavía se encarnizaron contra Alda: le infligieron una pena de 5 años de destierro en las Islas Tremiti y después en Corleto Perticara en la Región de Basilicata. De ese período oscuro de la vida de Alda nos informa Juares Busoni ${ }^{32}$, testigo directo que también estaba expiando el destierro en las Isole Tremiti. El viaje empezó en Ferrara y luego siguió hacia Ancona, Pescara y Foggia: cuando los presos fueron llevados hacia el tren, Alda iba marchando tranquila; otros compañeros la reconocieron y la invitaron muy amablemente a subir al tren delante de ellos como para desviarla a la mirada investigadora de la gente. Busoni recuerda acontecimientos tristes, escuchados de viva voz de Alda: el sucio dormitorio en la cárcel de Foggia, la convivencia con mujeres malhechoras, condenadas por crímenes comunes, la larga y extenuante espera del ferry en Manfredonia, la mar agitada y el miedo del futuro.

Una vez llegados a la isla de San Nicola, Costa y Busoni tuvieron que separarse: todas las mujeres tenían que vivir en la isla de San Domino para después embarcarse hasta el destino sucesivo. Alda tuvo la oportunidad de conocer a los otros antifascistas ${ }^{33}$ y había escrito a su hermana que, aunque en ausencia, estaba volviendo a pensar y a debatir: la Jefatura de policía de Foggia interceptó la carta y Alda fue trasladada a la Región de Basilicata donde pasó sus últimos angustiosos meses de destierro. Le redujeron la condena a dos años, pero su vuelta a Ferrara no produjo reintegración alguna al empleo: Alda tuvo que pedir el retiro adelantado y vivir como una reclusa en casa de su cuñada, continuamente vigilada.

No obstante, ella desempeñó un papel importante en su intención de mantener las fuerzas de oposiciones unidas y de trabajar para la propaganda; en 1942 fue arrestada nuevamente y la detuvieron en la cárcel por un mes. Fue torturada por apoderarse de nombres y particulares de la lucha contra el fascismo.

El ocho de septiembre la perdonaron pero otros acontecimientos más significativos determinaron su fin. En Ferrara, en un ajuste de cuentas por el poder dentro de los Repubblichini, Iginio Ghibellini, del cuerpo federal, fue

31 ASCFe, sobre n. 25, fasc. I, Causa Alda Costa-Municipio di Ferrara, suspensión del ejercicio de la profesión, 17 de marzo de 1926.

32 JUARES BUSONI, Nel tempo del fascismo, Roma, Ed. Riuniti, 1975. Se describe Alda como una mujer "con una sonrisa infantil en la cara no tan joven pero aún fresca, iluminada por dos ojos inteligentes y vivos" (p. 111).

33 Sobre la personalidad durante el destierro véase CELSO GHINI, ADRIANO DAL PONT, Gli antifascisti al confino 1926-1943, Roma, Editori Riuniti, 1971, y RENATO SITTI, Antifascisti ferraresi al tribunale speciale e al confino, Ferrara, Cartografia artigiana, 1983. 
asesinado y la represalia fue terrible: en la famosa larga noche entre el 14 y el 15 de noviembre de 1943 fueron arrestadas 79 personas, de las cuales 11 fusiladas ${ }^{34}$. Los hebreos presos fueron exiliados. Alda Costa fue encarcelada en Copparo $^{35}$ : debido a sus serísimas condiciones de salud fue trasladada al hospital de Copparo donde murió a las 2.30 de la tarde del 30 de abril de 1944 por "leucemia linfática crónica" a la edad de 68 años. A ella no se le concedió tampoco el entierro porque se temían represalias y tumultos por parte de aquellos que habrían querido rendirle homenaje.

Creo que es mi deber terminar ese recuerdo de la persona de Alda con sus mismas palabras que todavía suenan actuales y llenas en enseñanzas morales y coherencia:

Mi conciencia está tranquila hoy también, segura de baber desempeñado escrupulosamente mi deber de educadora, de mujer y ciudadana Italiana, sin nunca disimular mi pensamiento ni tampoco mi acción ${ }^{36}$.

En esa admirable síntesis podemos reconocer la historia de una vida entera entregada a la educación de los últimos y a la abnegación en sí.

34 También ese trágico acontecimiento permanence en la memoria histórica de Ferrara; véase el cuento de GIORGIO BASSANI, Una notte del '43, en Dentro le mura, Il romanzo di Ferrara I, Milano, Mondadori, 1973, pp. 239-295, y la película del director cinematográfico de la ciudad de Ferrara Florestano Vancini, La lunga notte del '43 (1960). Para más detalles véase DELFINA TROMBONI, Ferrara, 15 novembre 1943, en ALDO BERSELLI, op. cit., p. 265.

35 Cfr. GIANNA VANCINI, SANDRO FERRANTI, DARIO AMBROSONE, Le Carceri Mandamentali nella storia di Copparo e del suo territorio, Copparo, Edizioni del Comune di Copparo, 2004; en las pp. 13-23 se recuerda a Alda Costa.

36 ASCFe, sobre 25, fasc.I, Causa Alda Costa- Municipio di Ferrara, carta de Alda Costa al alcalde de Ferrara, 19 de marzo de 1926. 
